\title{
Trypanosoma cruzi: amastigote polymorphism defined by monoclonal antibodies
}

N.V. Verbisck,

S. Da-Silva and

R.A. Mortara
Departamento de Microbiologia, Imunologia e Parasitologia, Escola Paulista de Medicina, Universidade Federal de São Paulo, São Paulo, SP, Brasil

\section{Correspondence}

R.A. Mortara

Departamento de Microbiologia,

Imunologia e Parasitologia

EPM, UNIFESP

Rua Botucatu, 862, 60 andar

04023-062 São Paulo, SP

Brasil

Fax: + 55-11-571-1095

E-mail: renato.dmip@epm.br

Part of a Master's thesis

presented by N.V. Verbisck to the

Departamento de Microbiologia,

Imunologia e Parasitologia, EPM,

UNIFESP, São Paulo, SP, Brasil.

Research supported by CAPES,

PADCT (No. II 62.0247/94.0 SBIO),

UNDP/W orld Bank/W HO Special

Programme for Research and Training

in Tropical Diseases, and the

Rockefeller Foundation (No. 880277).

N.V. Verbisck and R.A. M ortara are

recipients of FAPESP fellowships

(Nos. 93/3918-3 and 94/0558-9/

94/3496-4, respectively).

$\ldots \ldots \ldots \ldots \ldots \ldots \ldots$

Received March 26, 1998

Accepted September 3, 1998

\section{Abstract}

We have raised monoclonal antibodies (mAbs) directed towards amastigote forms of Trypanosoma cruzi, and shown that mAbs 1D9 and 4B9 are carbohydrate while mAb 4B5 activity is resistant to periodate oxidation of the antigen. Here we used an ELISA to quantitate and compare the expression of surface epitopes on fixed parasites among different parasite isolates. The expression of markers varied among $T$. cruzi amastigotes isolated from infected cells or after extracellular differentiation of trypomastigotes. Moreover, we also observed an extensive polymorphic expression of these epitopes among amastigotes derived from different strains and clones. For instance, mAb 2C2 strongly and evenly reacted with 9 strains and clones (G, Y, CL, Tulahuen, MD, and F, and clones Sylvio X-10/4, D11, and CL.B), with absorbance at $492 \mathrm{~nm}\left(\mathrm{~A}_{492} \mathrm{~nm}\right)$ from 0.6 to 0.8 . By contrast, mAb 4B5 had a higher expression in Tulahuen amastigotes (around 0.9 at $492 \mathrm{~nm}$ ) whereas its reactivity with amastigotes from clones CL.B, Sylvio X-10/4 and D11 was much lower (around $0.4)$. mAb 1D9 displayed an interesting pattern of reactivity with amastigotes of the different strains and clones $\left(\mathrm{A}_{492} \mathrm{~nm}\right.$ of $\mathrm{G}>\mathrm{D} 11 \geq$ Sylvio $\mathrm{X}-10 / 4=\mathrm{MD}>$ Tulahuen $=\mathrm{F}=\mathrm{Y}>\mathrm{CL}>\mathrm{CL}$. B). Finally, we observed that $\mathrm{mAb} 4 \mathrm{~B} 9$ had the lowest reaction with the parasites studied, with higher values of $\mathrm{A}_{492} \mathrm{~nm}$ with $\mathrm{Y}$ strain (around 0.6 ) and lower values with Tulahuen, F and CL.B strains (around 0.2). Immunoblotting analysis also showed extensive variations among amastigotes of the various parasite isolates and mAbs 4B9, 1D9 and 4B5 revealed significant differences in expression between clones and parental strains. These data describe a previously uncharacterized polymorphism of $T$. cruzi amastigote surface components.

\section{Introduction}

Trypanosoma cruzi, the causative agent of Chagas' disease, exists in nature as a complex and highly variable population (1). Thus, a variety of strains and clones isolated and used by different laboratories display very distinct biological properties (1-3). Prop-

\section{Key words}

- Trypanosoma cruzi

- Amastigote

- Monoclonal antibodies

- Surface antigen

- Polymorphism erties such as tissue tropism (4), resistance to complement lysis (5), virulence (6), and infectivity in different animal models (7) were extensively examined using either metacyclic or tissue culture-derived trypomastigotes, regarded as the classic infective form of the parasite. Several studies attempting to correlate strain and clone polymorphism with bio- 
logical properties of trypomastigotes have implicated specific surface antigens (8-12). Tissue culture-derived trypomastigotes may differentiate extracellularly into amastigotes that resemble the proliferative intracellular forms in many aspects (13). T. cruzi amastigotes have also been shown to be infective, both for phagocytic (14-16) and non-phagocytic cells $(14,17,18)$. Although it has been reported that amastigotes express specific membrane components that might be involved in their uptake by phagocytes $(16,19)$, the parasite molecules involved in cell invasion, particularly of non-professional phagocytes, are still unknown. Detailed work by Andrews et al. $(13,20)$ showed that both intracellular and extracellular forms express a major surface glycoprotein designated Ssp4 that is bound to the membrane via a GPI anchor. Another T. cruzi amastigote-specific component named amastin was recently isolated by Teixeira et al. (21). Although its relation to Ssp-4 is unclear at the moment, the analysis of the amastin sequence indicates that it is a hydrophobic polypeptide lacking the characteristic GPI-anchor sequence (22) that would be expected for Ssp4. Other amastigote specific antigens that have recently been cloned and sequenced based on previously published amino-terminal sequence data (23) were shown to belong to the trans-sialidase/sialidase superfamily (24). In the present study we show that $T$. cruzi amastigotes from distinct strains and clones express different epitopes in a polymorphic manner, similar to that described for epitopes defined for other parasite stages.

\section{Material and Methods}

\section{Parasites and cells}

Extracellular amastigotes (EA) from $T$. cruzi G (25), Y (26), CL (27), CL.B clone (28), MD (provided by Maria P. Deane from Instituto Oswaldo Cruz, Rio de Janeiro, RJ, Brasil), F (29), Sylvio X-10/4 clone (30) and
Tulahuen (31) strains were isolated after differentiation of tissue culture-derived trypomastigotes in LIT medium as previously described (13) and intracellular (IA) parasites were purified from infected Vero cells after mechanical disruption and centrifugation through a $10 / 15 \%$ metrizamide gradient (32). We also examined, for comparison, amastigotes isolated from supernatants of infected Vero cells, which were designated tissue culture amastigotes (TCA). In several experiments we used a clone, designated D1 1, isolated in our laboratory from $\mathrm{G}$ strain by the method described by Lima and Villalta (33), and the clone CL.B. Some characteristics of the D11 clone resemble the parental strain. D11 causes subpatent parasitemias in outbred albino mice, and metacyclic trypomastigotes displaying the monoclonal antibody (mAb) 1G7-reactive antigen, gp90, are completely lysed by this mAb $(10,34)$.

Vero cells (obtained from Instituto Adolfo Lutz, São Paulo, SP, Brasil) were cultured in RPMI-1640 medium supplemented with $10 \%$ fetal bovine serum (FBS, Cultilab, Campinas, SP, Brasil), $10 \mu \mathrm{g} / \mathrm{ml}$ streptomycin, 100 $\mathrm{U} / \mathrm{ml}$ penicillin, and $40 \mu \mathrm{g} / \mathrm{ml}$ gentamycin at $37^{\circ} \mathrm{C}$ in a $5 \% \mathrm{CO}_{2}$ humid atmosphere. Cells were subcultured every $2 / 3$ days and after infection they were kept in RPMI $/ 2 \%$ FBS at $33^{\circ} \mathrm{C}$ to improve the yield of released trypomastigotes.

\section{Monoclonal antibodies}

mAbs were produced by immunizing $\mathrm{BALB} / \mathrm{c}$ mice with amastigotes from clone D11 of the $G$ strain. Their isolation and immunochemical characterization have been described elsewhere (35). Briefly, mAb 4B5 (IgG1) was obtained from mice immunized with psoralen/UV-inactivated (36) intracellular amastigotes, and mAbs 1D9 (IgG3) and 4B9 (IgG3) were isolated after immunization with mixtures of heat-inactivated intracellular and extracellular amastigotes of the same clone D11. 
All antibodies were used as ascitic fluids previously titrated onto fixed parasites (see below) before routine use to determine the highest dilution that provided maximum absorbance at $492 \mathrm{~nm}$ (1:200 for anti-p30, 1D9, 4B5, and 4B9; 1:1000 for 2C2). mAb $2 \mathrm{C} 2(\operatorname{IgG} 2 \mathrm{a})$, which is specific for the stagespecific $84-\mathrm{kDa}$ glycoprotein of $T$. cruzi amastigotes (13), was kindly provided by Norma Andrews (Yale University School of Medicine, New Haven, CT, USA), and mAb anti-p30 (IgG2b), specific for a 30-kDa protein of L. (L.) amazonensis amastigotes (37), was kindly provided by Clara L. Barbiéri (Universidade Federal de São Paulo, São Paulo, SP, Brasil).

\section{ELISA on glutaraldehyde-fixed amastigotes}

Isolated parasites were washed 3 times in phosphate-buffered saline (PBS) and $50 \mu 1$ containing $1 \times 10^{6}$ parasites was added to the wells of ELISA strip plates (Costar, medium or high binding capacity) and centrifuged at $800 \mathrm{~g}$ for $5 \mathrm{~min}$. The supernatant was carefully removed by aspiration and the parasites were fixed with $0.25 \%(\mathrm{v} / \mathrm{v})$ glutaraldehyde (Electron Microscopy Sciences, Fort Washington, PA, USA) in PBS at room temperature for $20 \mathrm{~min}$. After 4 washes in PBS to remove excess glutaraldehyde, the wells were blocked for at least $12 \mathrm{~h}$ at $4^{\circ} \mathrm{C}$ with $5 \%$ defatted powdered milk in PBS $/ 0.05 \%$ sodium azide (PBS-M). At this stage, plates could be stored at $-20^{\circ} \mathrm{C}$ for at least three months. Prior to use, the plates were warmed to room temperature for all subsequent steps: the PBS/milk was removed and the parasites were incubated with $50 \mu 1$ of the antibodies diluted 1:50 in PBS-M for $1 \mathrm{~h}$. After 3 washes with PBS, the bound Ig was developed by incubation with anti-mouse Ig coupled to horseradish peroxidase (Sigma Chemical Co., St. Louis, MO, USA) diluted 1:2000 (titrated dilution) in PBS-M. After 1 $\mathrm{h}$ the conjugate was washed 3 times with PBS and $50 \mu \mathrm{l}$ of substrate solution $(10 \mathrm{mg}$ $o$-phenylenediamine (OPD) diluted in $25 \mathrm{ml}$ $0.16 \mathrm{M}$ citrate-phosphate buffer, $\mathrm{pH}$ 5.0, plus $10 \mu \mathrm{l}$ of $30 \% \mathrm{H}_{2} \mathrm{O}_{2}$ ) was added to each well as the substrate for the reaction. The enzymatic reaction was stopped after $4 \mathrm{~min}$ with $4 \mathrm{~N} \mathrm{H}_{2} \mathrm{SO}_{4}$, and absorbance at $492 \mathrm{~nm}$ was determined with a Multiskan MS 352 reader (Labsystems, Helsinki, Finland). All readings were made in quintuplicate for each determination, in at least three independent experiments.

\section{ELISA standardization}

In order to assess the reliability, sensitivity and reproducibility of the ELISA in evaluating differences in the levels of expression of amastigote surface antigens, we performed several controls. First, we determined that a variation of up to $15 \%$ in the number of parasites/well did not significantly (less than $10 \%$ in absorbance values) influence the final values (Figure 1). This ensured that possible errors in parasite counting did not affect the final absorbance values. Second, we repeated the experiments with the different antibodies several times, using stored or freshly prepared fixed-amastigote plates, and

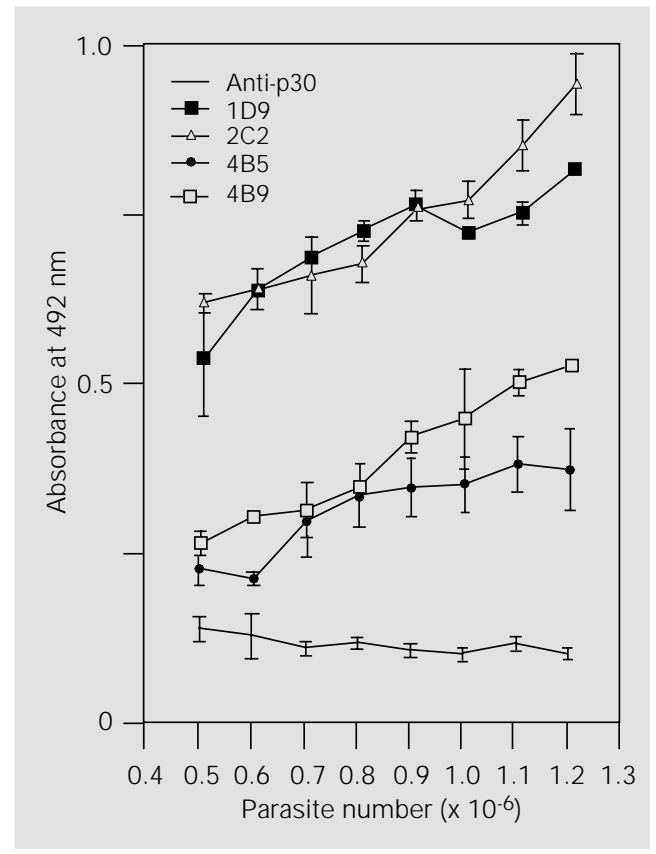

Figure 1 - ELISA absorbance is not substantially altered with differences up to $15 \%$ in the number of parasites. Extracellular amastigotes from clone D11 were obtained in LIT medium after $48 \mathrm{~h}$ of incubation, counted and dispensed in increasing quantities in strip wells for ELISA with mAbs 1D9, 2C2, 4B5, 4B9 and anti-p30 (the last one used as a negative control). Each point indicates the mean plus one standard deviation for a triplicate determination. 
Figure 2 - Expression of epitopes defined by mAbs 1D9, 2C2, 4B5 and $4 \mathrm{~B} 9$ is variable among amastigotes obtained from different sources. Amastigotes from clone D11 were isolated from infected cells (Intra), LIT medium after $48 \mathrm{~h}$ (Extra) or infected Vero cell supernatants (TCA), and studied by ELISA. Bars indicate mean value plus one standard deviation for a quintuplicate determination. mAb anti-p30 was used as a negative control.

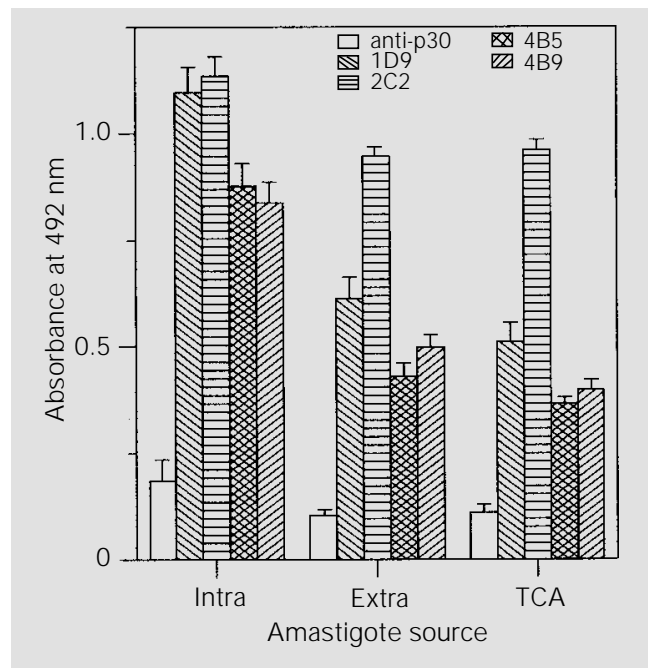

always obtained consistent results where the relative values of absorbance of the different mAbs were the same in experiments carried out up to two months apart (data not shown). Third, the antibodies always retained their specificity and heterologous reagents always gave background values when applied to fixed amastigotes (data not shown). Fourth, using ELISA, we confirmed the results of Andrews et al. (20), who reported that the surface expression of Ssp-4 antigen on extracellular amastigotes is high after $24 \mathrm{~h}$ of differentiation and decreases if parasite culture in LIT medium proceeds for up to $72 \mathrm{~h}$. However, the signal of an irrelevant $\mathrm{mAb}$ was not altered during this period (data not shown). The reduction in the expression of surface Ssp-4 is accounted for by phospholipase cleavage of the GPI anchor of the molecule, releasing soluble Ssp-4 into the culture medium (20).

\section{Protein gel electrophoresis and immunoblotting}

Sodium dodecyl sulfate electrophoresis (38) and immunoblotting (39) of protein amastigote extracts were performed on $10 \%$ resolving gels using a mini-PROTEAN ${ }^{\circledR}$ II electrophoresis cell and a mini-trans-blot module (Bio-Rad Laboratories, Hercules, CA, USA), as previously described $(10,35)$.

\section{Results}

\section{Surface expression of amastigote epitopes is variable among different T. cruzi strains and clones}

We established that our ELISA could reliably identify differences in the surface expression of epitopes defined by our mAbs. The nature of the epitopes recognized by mAbs 1D9, 4B5 and 4B9 was determined previously (35). Briefly, $10 \mathrm{mM}$ sodium mperiodate treatment of blots containing protein amastigote ( $\mathrm{G}$ strain) extracts abolished immunoblot reactivity of mAbs 1D9 and $4 \mathrm{~B} 9$ but not 4B5, suggesting that only the last $\mathrm{mAb}$ may recognize a protein moiety, while the other two recognize carbohydrate epitopes. The observations that L-fucose inhibits the binding of $2 \mathrm{C} 2$ to glutaraldehydefixed amastigotes in ELISA indicate that the corresponding epitope may also be a carbohydrate in nature (Verbisck NV, Da-Silva S and Mortara RA, unpublished data).

We next determined the relative expression of the epitopes recognized by mAbs 1D9, 2C2 and 4B5 in IA and EA isolated from either culture supernatants (TCA) or LIT medium (Extra). Figure 2 shows that, unlike $\mathrm{mAb} 2 \mathrm{C} 2$ that is expressed abundantly in all forms, the epitopes recognized by mAbs 1D9, 4B9 and 4B5 were considerably more abundant in intracellular parasite form.

We subsequently examined the reactivities of the mAbs in ELISA, using extracellular amastigotes of different strains. The results are summarized in Figure 3 and indicate that the mAbs can detect substantial inter-strain variation in their relative expressions. Several interesting features emerge from the analysis of these results: i) the epitope identified by 1D9 (Figure 3A) was scarce in the $\mathrm{Y}$ and CL strains, and present in intermediate amounts in MD, Tulahuen, and F amastigotes; ii) the epitope recognized by $\mathrm{mAb} 2 \mathrm{C} 2$ was more evenly represented in all 
strains (Figure 3B); iii) the mAb 4B5 target was more abundant in Tulahuen, $\mathrm{G}$, and $\mathrm{CL}$ parasites and was moderately expressed in $\mathrm{Y}$ and $\mathrm{MD}$ isolates (Figure 3C); iv) the $\mathrm{mAb}$ 4B9 epitope was poorly expressed in Tulahuen and CL parasites but was more abundant in $\mathrm{MD}, \mathrm{G}$ and $\mathrm{Y}$ parasites (Figure 3D). We also observed with some mAbs marked differences in epitope expression between clones and parental strains. For instance, whereas $2 \mathrm{C} 2$ expression by CL.B was higher than $\mathrm{CL}$ expression, the opposite pattern was observed with the other epitopes which were more abundant in the parental strain (Figure 3). Similarly, epitopes recognized by mAbs $4 \mathrm{~B} 9$ and $2 \mathrm{C} 2$ were equally abundant in clone D1 1 and in the G strain but mAbs 1D9 and 4B5 had a higher expression in the parental strain (Figure 3).

\section{Expression of amastigote antigens was polymorphic among T. cruzi isolates}

Immunoblotting analysis using $\mathrm{mAbs}$ 1D9, 2C2, 4B5, and 4B9 revealed a polymorphic pattern of antigen expression among the different strains and clones. As shown in Figure 4, several bands present in some isolates were clearly not detected in others (e.g., 1D9 in CL, Y strains), while the relative molecular masses may also vary to a substantial extent (e.g., 4B5 in G, Sylvio X-
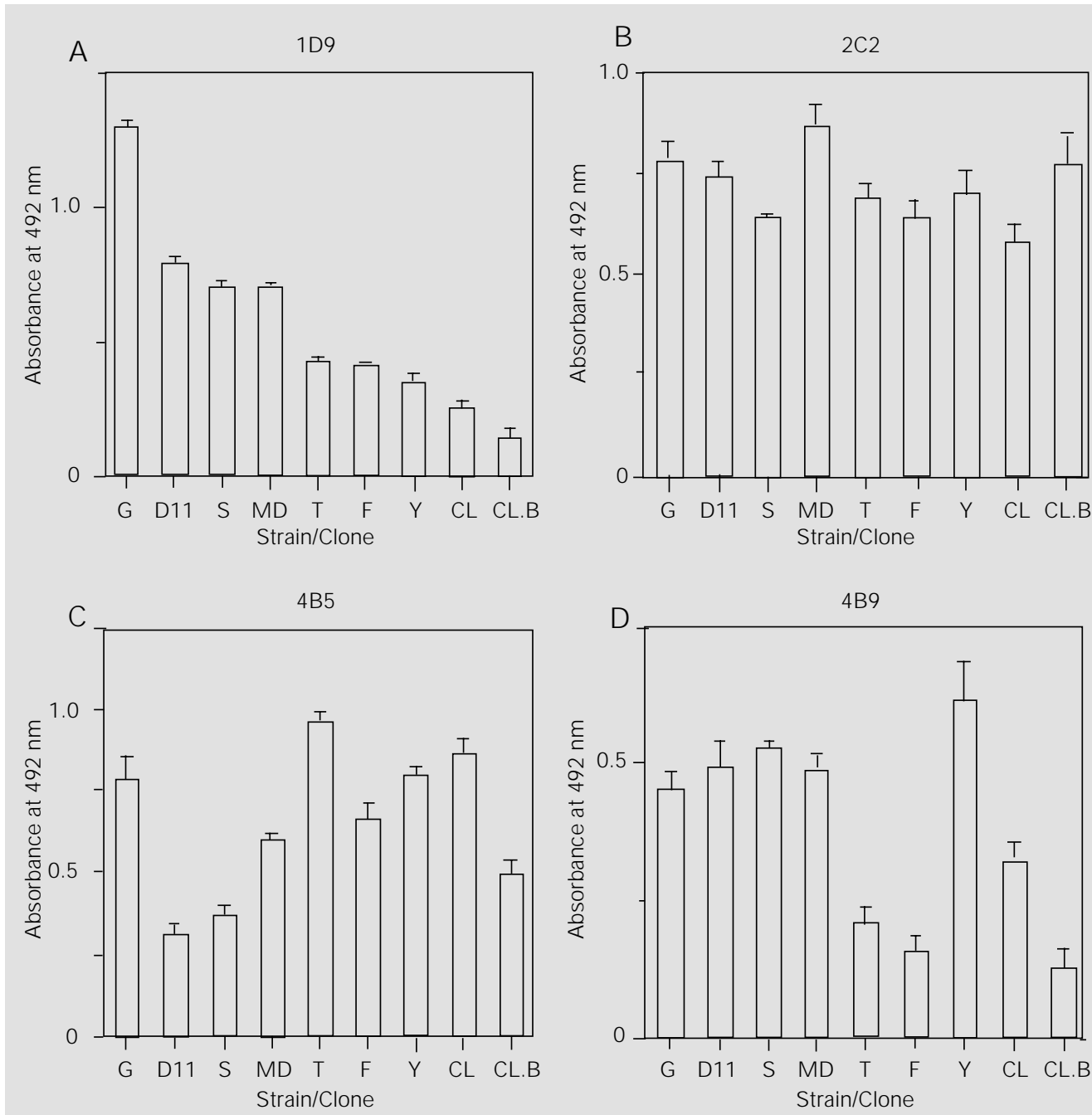

Figure 3 - Expression of epitopes defined by mAbs 1D9, 2C2, 4B5 and $4 \mathrm{~B} 9$ is variable among amastigotes of different T. cruzi strains and clones. Extracellular amastigotes were obtained in LIT medium after a 48-h incubation and analyzed by ELISA. Bars indicate the mean subtracted from the background values obtained with mAb anti-p30 (always lower than 0.2 ) plus one standard deviation for a quintuplicate determination. 
$10 / 4$, and F strains). However, the absence of immunoblot reaction in some strains that give positive reaction by ELISA (e.g., 1D9 reaction with Y, CL and CL.B) can be explained by the behavior of this $\mathrm{mAb}$ under different experimental conditions, since this antibody is still capable of immunoprecipitating an $84-\mathrm{kDa}$ component or to surface label Y and CL (but not CL.B) extracellular amastigotes by immunofluorescence (35). In a similar way, mAb $2 \mathrm{C} 2$ immunoprecipitated an $84-\mathrm{kDa}$ major component of $\mathrm{G}$, D11, Sylvio X-10/4 and CL.B extracellular amastigotes despite its poor or absent reaction with these parasites in immunoblot (data not shown; Figure 4). Conversely, immunoblot reaction of mAb 4B5 was not observed in ELISA, e.g., the poor reaction with D11, CL.B and Sylvio X-10/4. This apparent lack of correlation could be explained if we assume that there is a significant reaction of this mAb with D11 cytoplasmic components of amastigotes as shown by immunofluorescence and immunoelectronmicroscopy (35).

\section{Discussion}

Amastigotes obtained by axenic differentiation of tissue culture trypomastigotes contain a major $84-\mathrm{kDa}$ iodinatable glycoprotein designated Ssp-4 that is linked to the parasite membrane through a GPI anchor $(13,20)$. Ssp-4 and other structurally related components are the parasite's immunodominant elements since they are readily recognized by different $\mathrm{mAbs}$ raised against isolated amastigotes $(13,23,35,40)$. In the present study we have described a previously uncharacterized polymorphism of $T$. cruzi amastigote surface components. Using mAbs directed at carbohydrate and non-carbohy-
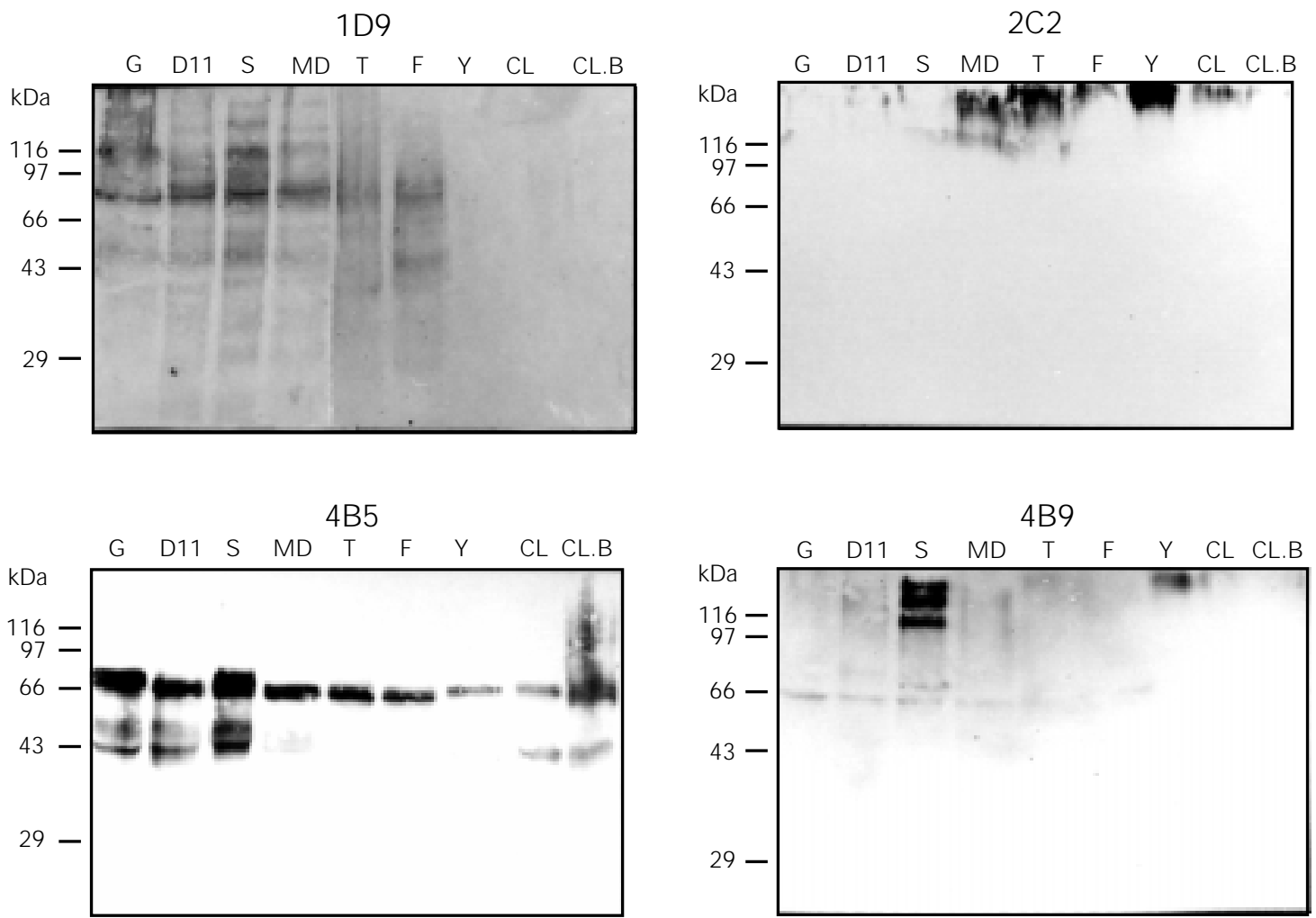

Figure 4 - Reactivity of mAbs 1D9, 2C2, 4B5 and 4B9 with amastigote polypeptides is polymorphic among different T. cruzi strains and clones. Extracellular amastigote (LIT medium) extracts were processed for immunoblotting as described elsewhere (35). Apparent molecular masses in kilodaltons are indicated on the left of each panel. 
drate epitopes located on the parasite surface, we could detect by ELISA both strain and clonal variation in the expression of these epitopes among amastigotes of different $T$. cruzi isolates. In addition, immunoblotting analysis of these parasites using the same mAbs revealed particular patterns of reaction, indicating a polymorphism in the expression of these antigens among the different strains and clones of T. cruzi. A similar behavior regarding distinct reactivities of $\mathrm{mAbs}$ in different immunochemical assays (e.g., immunofluorescence, immunoblotting, complement-mediated lysis and agglutination) has been extensively documented in the literature. We have previously described a similar trend in the expression of a mucin and of a major surface component of $T$. cruzi metacyclic trypomastigotes $(10,11)$.

We have observed that most of the mAbs that recognize Ssp-4 react with carbohydrate epitopes (35), a fact that has facilitated the use of glutaraldehyde fixation in our ELISAs. Indeed, a survey of the literature shows that most of the mAbs raised against T. cruzi amastigotes recognize carbohydrate epitopes $(13,23)$. These observations suggest that these developmental stages are covered with a significant proportion of sugar residues. Polymorphism of surface molecules seems to be a common feature of $T$. cruzi forms and has been described in epimastigotes (3), tissue culture trypomastigotes $(8,9)$ and metacyclic trypomastigotes $(10,11)$. If the polymorphic expression of peptide epitopes in these studies has emerged as a fairly common feature for $T$. cruzi $(3,8,9)$, the variability in the carbohydrate moieties mainly attached to parasite surface glycoproteins is less well characterized (11). Other parasitic protozoans like Plasmodium and Leishmania have also been shown to display extensive antigenic polymorphism $(41,42)$.

There are numerous strains and clones of T. cruzi being used by different laboratories. These strains have distinct biological prop- erties. Some isolates can even contain subpopulations that range from highly lethal to mildly infective in murine models (30). Although the molecular basis for this diversity is not known, it is likely that chromosomal rearrangements also occur in $T$. cruzi, similar to what has been described for other protozoans like Giardia, T. brucei and Plasmodium (43).

Extracellular amastigotes of the $\mathrm{Y}$ strain have been shown to be infective for different cell types and are able to sustain an infective cycle in vivo (14). Comparative studies carried out with both intracellular and extracellular forms of the Y strain indicate that extracellular amastigotes are more infective and comparatively more resistant to complementmediated lysis (Barros HC, Verbisck NV, Da-Silva $\mathrm{S}$ and Mortara RA, unpublished data). Although several studies have shown that amastigotes can be found in the peripheral blood of infected animals $(14,44)$, particularly during the acute phase of the disease, the role of circulating amastigotes in the course of natural infection remains unclear $(14,19)$. T. cruzi strains that are particularly virulent and lethal for animal models always induce high parasitemias, a fact that has been correlated with the infectivity of the circulating trypomastigotes. In preliminary studies, we have found that extracellular amastigotes from $T$. cruzi strains that are less virulent and only cause subpatent parasitemia, like the $\mathrm{G}$ and MD strains, are far more infective to HeLa cells than forms of the more virulent isolates like Tulahuen, F, Y and CL strains (Barros HC, Da-Silva S and Mortara RA, unpublished data). Whether this unexpected pattern of infectivity is significant in the natural course of infection in vivo is not known, but this phenomenon clearly deserves further investigation. Since Ssp-4 is a major surface component of $T$. cruzi amastigotes it could also play a role in the process of parasite adhesion and invasion. 


\section{References}

1. McDaniel J P \& Dvorak J A (1993). Identification, isolation, and characterization of naturally-occurring Trypanosoma cruzi variants. Molecular and Biochemical Parasitology, 57: 213-222.

2. Macedo AM, Martins MS, Chiari E \& Pena SDJ (1992). DNA fingerprinting of Trypanosoma cruzi: A new tool for characterization of strains and clones. Molecular and Biochemical Parasitology, 55: 147-154.

3. Flint J E, Schechter M, Chapman MD \& Miles MA (1984). Zymodeme and species specificities of monoclonal antibodies raised against Trypanosoma cruzi. Transactions of the Royal Society of Tropical Medicine and Hygiene, 78: 193-202.

4. Araujo-j orge TC, Barbosa HS, Moreira AL, De Souza W \& Meirelles MNL (1986). The interaction of myotropic and macrophagotropic strains of Trypanosoma cruzi with myoblasts and fibers of skeletal muscle. Zeitschrift für Parasitenkunde, 72: 577584.

5. J acobson KC, Washburn RG \& Kuhn RE (1992). Binding of complement to trypomastigotes of a Brazilian strain of Trypanosoma cruzi: Evidence for heterogeneity within the strain. J ournal of Parasitology, 78: 697-704.

6. Andrade SG (1990). Influence of Trypanosoma cruzi strain on the pathogenesis of chronic myocardiopathy in mice. Memórias do Instituto Oswaldo Cruz, 85: 17-27.

7. Rottenberg ME, Riarte A, Sporrong L, Altcheh J , Petray P, Ruiz AM, Wigzell $H$ \& Örn A (1995). Outcome of infection with different strains of Trypanosoma cruzi in mice lacking CD4 and/or CD8. Immunology Letters, 45: 53-60.

8. Abuin G, Colli W, De Souza W \& Alves MJ M (1989). A surface antigen of Trypanosoma cruzi involved in cell invasion (TC85 ) is heterogeneous in expression and molecular constitution. Molecular and Biochemical Parasitology, 35: 229-237.

9. Prioli RP, Mejia J S \& Pereira MEA (1990). Monoclonal antibodies against Trypanosoma cruzi neuraminidase reveal enzyme polymorphism, recognize a subset of trypomastigotes, and enhance infection in vitro. J ournal of Immunology, 144: 43844391.

10. Mortara RA, Araguth MF \& Yoshida N (1988). Reactivity of stage-specific monoclonal antibody $1 \mathrm{G} 7$ with metacyclic trypomastigotes of Trypanosoma cruzi strains: lytic property and antigen polymorphism. Parasite Immunology, 10: 369378.
11. Mortara RA, Da-Silva S, Araguth MF, Blanco SA \& Yoshida N (1992). Polymorphism of the 35- and 50-kiloDalton surface glycoconjugates of Trypanosoma cruzi metacyclic trypomastigotes. Infection and Immunity, 60: 4673-4678.

12. Schenkman S, Ferguson MAJ, Heise N, Cardoso de Almeida ML, Mortara RA \& Yoshida N (1993). Mucin-like glycoproteins linked to the membrane by glycosylphosphatidylinositol anchor are the major acceptors of sialic acid in a reaction catalyzed by trans-sialidase in metacyclic forms of Trypanosoma cruzi. Molecular and Biochemical Parasitology, 59: 293304.

13. Andrews NW, Hong $K$, Robbins ES \& Nussenzweig V (1987). Stage-specific surface antigens expressed during the morphogenesis of vertebrate forms of Trypanosoma cruzi. Experimental Parasitology, 64: 474-484.

14. Ley V, Andrews NW, Robbins ES \& Nussenzweig $\vee$ (1988). Amastigotes of Trypanosoma cruzi sustain an infective cycle in mammalian cells. J ournal of Experimental Medicine, 168: 649-659.

15. McCabe RE, Remington J $S$ \& Araujo FG (1984). Mechanisms of invasion and replication of the intracellular stage in Trypanosoma cruzi. Infection and Immunity, 46: 372-376.

16. Noisin EL \& Villalta F (1989). Fibronectin increases Trypanosoma cruzi amastigote binding to and uptake by murine macrophages and human monocytes. Infection and Immunity, 57: 1030-1034.

17. Mortara RA (1991). Trypanosoma cruzi: amastigotes and trypomastigotes interact with different structures on the surface of HeLa cells. Experimental Parasitology, 73: 1-14.

18. Pan SC-T (1978). Trypanosoma cruzi: In vitro interactions between cultured amastigotes and human skin-muscle cells. Experimental Parasitology, 45: 274-286.

19. Kahn S, Wleklinski M, Aruffo A, Farr A, Coder D \& Kahn M (1995). Trypanosoma cruzi amastigote adhesion to macrophages is facilitated by the mannose receptor. J ournal of Experimental Medicine, 182: 1243-1258.

20. Andrews NW, Robbins ES, Ley V, Hong KS \& Nussenzweig $\vee$ (1988). Developmentally regulated, phospholipase C-mediated release of the major surface glycoprotein of amastigotes of Trypanosoma cruzi. J ournal of Experimental Medicine, 167: 300-314.
21. Teixeira SMR, Russell DG, Kirchhoff LV \& Donelson J E (1994). A differentially expressed gene family encoding "amastin", a surface protein of Trypanosoma cruzi amastigotes. J ournal of Biological Chemistry, 269: 20509-20516.

22. Cross GAM (1990). Glycolipid anchoring of plasma membrane proteins. Annual Review of Cell Biology, 6: 1-39.

23. Pan AA \& McMahon-Pratt D (1989). Amastigote and epimastigote stage-specific components of Trypanosoma cruzi characterized by using monoclonal antibodies. J ournal of Immunology, 143: 1001-1008.

24. Low HP \& Tarleton RL (1997). Molecular cloning of the gene encoding the $83-\mathrm{kDa}$ amastigote surface protein and its identification as a member of the Trypanosoma cruzi sialidase superfamily. Molecular and Biochemical Parasitology, 88: 137-149.

25. Yoshida N (1983). Surface antigens of metacyclic trypomastigotes of Trypanosoma cruzi. Infection and Immunity, 40: 836-839.

26. Silva LHP \& Nussenzweig V (1953). Sobre uma cepa de Trypanosoma cruzi altamente virulenta para o camundongo branco. Folia Clinica Biologica, 20: 191203.

27. Brener Z \& Chiari E (1963). Variações morfológicas observadas em diferentes amostras de Trypanosoma cruzi. Revista do Instituto de Medicina Tropical de São Paulo, 5: 220-224.

28. Cano MI, Gruber A, Vazquez M, Cortés A, Levin MJ, González A, Degrave W, Rondinelli $E$, Zingales $B$, Ramirez J L, Alonso C, Requena J M \& Da Silveira J F (1995). Molecular karyotype of clone CL Brener chosen for the Trypanosoma cruzi Genome Project. Molecular and Biochemical Parasitology, 71: 273-278.

29. Kloetzel J K \& Lafaille JJ (1983). Strain specific protective immunity against Trypanosoma cruzi. J ournal of Parasitology, 69: 267-270.

30. Postan M, Dvorak JA \& McDaniel JP (1983). Studies of Trypanosoma cruzi clones in inbred mice. I. A comparison of the course of infection of $\mathrm{C} 3 \mathrm{H} / \mathrm{HEN}$-mice with two clones isolated from a common source. American J ournal of Tropical Medicine and Hygiene, 32: 497-506.

31. Pizzi TP, Rubio MD, Prager R \& Silva RC (1952). Acción de la cortisona en la infección experimental por Trypanosoma cruzi. Boletin Chileno de Parasitologia, 7: 22-24.

32. Abrahamsohn IA, Katzin AM \& Milder RV 
(1983). A method for isolating Trypanosoma cruzi amastigotes from spleen and liver using two-step discontinuous gradient centrifugation. J ournal of Parasitology, 69: 437-439.

33. Lima MF \& Villalta F (1989). Trypanosoma cruzi trypomastigote clones differentially express a parasite cell adhesion molecule. Molecular and Biochemical Parasitology, 33: 159-170.

34. Yoshida N, Blanco SA, Araguth MF, Russo M \& Gonzalez J C (1990). The stage-specific 90-kilodalton surface antigen of metacyclic trypomastigotes of Trypanosoma cruzi. Molecular and Biochemical Parasitology, 39: 39-46.

35. Barros HC, Verbisck NV, Silva S, Araguth MF \& Mortara RA (1997). Distribution of epitopes of Trypanosoma cruzi amastigotes during the intracellular life cycle within mammalian cells. J ournal of Eukaryotic Microbiology, 46: 203-233.

36. Andrews NW, Alves MJ M, Schumacher RI \& Colli W (1985). Trypanosoma cruzi: protection in mice immunized with 8methoxypsoralen-inactivated trypomastigotes. Experimental Parasitology, 60: 255-
262.

37. Beyrodt CGP, Pinto AR, Freymuller E \& Barbiéri CL (1997). Characterization of an antigen from Leishmania amazonensis amastigotes able to elicit protective responses in a murine model. Infection and Immunity, 65: 2052-2059.

38. Laemmli UK (1970). Cleavage of structural proteins during the assembly of the head of bacteriophage T4. Nature, 227: 680-685.

39. Towbin H, Staehelin T \& Gordon J (1979). Electrophoretic transfer of proteins from polyacrylamide gels to nitrocellulose sheets: procedure and some applications. Proceedings of the National Academy of Sciences, USA, 76: 4350-4354.

40. lida $K \&$ Ley $\vee(1991)$. Isolation and characterization of a $92-\mathrm{KD}$ surface molecule of Trypanosoma cruzi amastigotes recognized by a monoclonal antibody that induces complement-mediated killing. American J ournal of Tropical Medicine and Hygiene, 45: 619-628.

41. Fenton B, Clark J T, Khan CMA, Robinson JV, Walliker D, Ridley RG, Scaife J G \& McBride JS (1991). Structural and anti- genic polymorphism of the 35- to 48kilodalton merozoite surface antigen (MSA-2) of the malaria parasite Plasmodium falciparum. Molecular and Cellular Biology, 11: 963-971.

42. Pimenta PFP, Saraiva EMB, Rowton E, Modi GB, Garraway LA, Beverley SM, Turco SJ \& Sacks DL (1994). Evidence that the vectorial competence of phlebotomine sand flies for different species of Leishmania is controlled by structural polymorphisms in the surface lipophosphoglycan. Proceedings of the $\mathrm{Na}$ tional Academy of Sciences, USA, 91: 9155-9159.

43. Lanzer $M$, Fischer $K \&$ Le Blancq SM (1995). Parasitism and chromosome dynamics in protozoan parasites: Is there a connection? Molecular and Biochemical Parasitology, 70: 1-8.

44. Morais Rêgo SF (1956). Sobre o encontro de formas tissulares do Trypanosoma cruzi (Chagas 1909) no sangue circulante do camundongo branco (Mus musculus). Folia Clínica Biológica, 26: 17-45. 


\section{Brazilian}

Journal of

Medical and

Biological

Research

on the

Internet

\section{Available at}

http://www.epub.org.br/bjmbr/

http://www.scielo.br/bjmbr.htm 